\title{
The Intrinsic Properties of the Local Interstellar Medium
}

\author{
Olivia Puyoo and Lotfi Ben Jaffel
}

Institut d'Astrophysique de Paris, 98 bis Blvd Arago, 75014 Paris, France

\begin{abstract}
We propose a new method to constrain the actual state of the interstellar cloud that surrounds the solar system. Using Voyager UVS Lyman- $\alpha$ sky maps and the powerful principle of invariance, we derive the $\mathrm{H}$ distribution all along the spacecraft path. Provided current models of the heliopause interface between the solar and the interstellar winds, we extrapolate this distribution to farther distances from the Sun and infer in a self consistent way key parameters of the local cloud. Our findings are a high interstellar hydrogen density of $\sim 0.24 \mathrm{~cm}^{-3}$ and a weak ionization $\frac{\mathrm{n}\left(\mathrm{H}^{+}\right)}{\mathrm{n}\left(\mathrm{H}^{+}\right)+\mathrm{n}(\mathrm{H})} \simeq 14 \%$.
\end{abstract}

\section{Introduction}

It is widely believed that the local interstellar medium represents a point sample of the more extended interstellar medium (ISM), and that its studies through its interplanetary signature is of particular interest to understand the origin and the evolution of the ISM. Because of the relative motion of the Sun with respect to the local interstellar cloud (LIC), the neutral component of the interstellar wind penetrates deeply inside the solar system, where it becomes accessible to in situ detections. After its discovery in the early 70's in the Earth neighborhood, interpretation of backscattered H Lyman- $\alpha$ and HeI $58.4 \mathrm{~nm}$ solar radiations has been extensively employed to derive $\mathrm{H}$ and He abundances in the local cloud. As classical interpretations of UV sky maps usually require the modeling of the $\mathrm{H}$ distribution in the heliosphere with the problem of filtration at the heliopause and the calculation of the radiation field including multi-scattering effects, the derivation of neutral abundances proved to be difficult, and the values found were consequently poorly constrained.

The aim of this paper is to present a new technique for deriving the intrinsic properties of the LIC by interpreting differently the UV backscattered radiation measurements. This method consists in constraining the $\mathrm{H}$ distribution stage by stage, starting from the Earth neighborhood up to the far unperturbed LIC. In a first step, we determine the $\mathrm{H}$ distribution along the Voyager trajectory by applying the principle of invariance to UVS Lyman- $\alpha$ sky maps recorded for several positions of the spacecraft (Puyoo et al 1997). Then we connect this distribution to the LIC one through a model of interaction between the solar and the interstellar flows. Lastly, assuming the LIC 
in a local steady state, we derive the intrinsic properties of the local cloud, i.e. the densities of both neutral and ionized hydrogen and helium in a self consistent way.

\section{Method}

Our technique is a continuation of a method recently proposed by Puyoo et al (1997) to derive in a self-consistent way the $\mathrm{H}$ neutral density in the inner heliosphere. This method is based on the invariance principle, which expresses, for a slab, the direct relationship between the radiation field incident on its boundaries and its optical properties, i.e. the reflexion and transmission coefficients and the contribution of inner sources. As the coefficients are dependent on the scattering gas distribution, the $\mathrm{H}$ distribution within the layer is therefore directly accessed. With that in view, we apply our technique to VOYAGER/UVS Lyman- $\alpha$ sky maps taken at different locations of the Spacecraft from 4 to $35 \mathrm{AU}$, which provides the $\mathrm{H}$ density distribution as shown in Fig. 1. It is worth to note that by using the principle of invariance, we avoided the modeling of the whole complex medium as it was previously required by classical techniques to interpret Voyager data. However, due to the local nature of our method, a limitation immediately appears as we can only constrain the density for regions visited by the Voyager spacecraft and for which Ly- $\alpha$ sky maps exist.

To reach larger distances from the Sun, we extrapolate the distribution shown in Fig. 1, using the sophisticated model of interaction between the solar wind and the supersonic flow as developed by Baranov \& Malama (1993). In such models, the filtration effects due to charge exchanges between interstellar hydrogen atoms and protons at the heliopause depend mainly on two parameters: the Mach number and the proton density of the LIC. In the following, we fix the Mach number at its commonly assumed value $M \simeq 2$ (Baranov \& Malama 1993) and consider the LIC proton density as a free parameter. We then extrapolate Voyager $\mathrm{H}$ distribution shown in Fig. 1 by varying both the LIC proton $\left(\mathrm{n}_{\mathrm{H}^{+}}\right)$and the $\mathrm{H}$ neutral $\left(\mathrm{n}_{\mathrm{H} \infty}\right)$ densities, which provides a first relation between the two parameters (see Fig. 2). A second relation is however necessary to simultaneously constrain them. For that end, we assume the unperturbed LIC to be in a local statistical equilibrium, and we solve the charge balance equations (e.g. Vallerga \& Welsh 1993) controlled by the ionization due to cosmic rays, known EUV stellar sources and the estimated contribution of the conductive interface between the local cloud and the surrounding local bubble (Slavin 1989). With the additional assumption of a cosmic ratio of $\mathrm{H} / \mathrm{He}=10$, we obtain a second relation between proton and $\mathrm{H}$ densities.

As shown in Fig. 2, this relation, combined with the one deduced through the Voyager distribution and the Baranov model, allows to derive in a self 


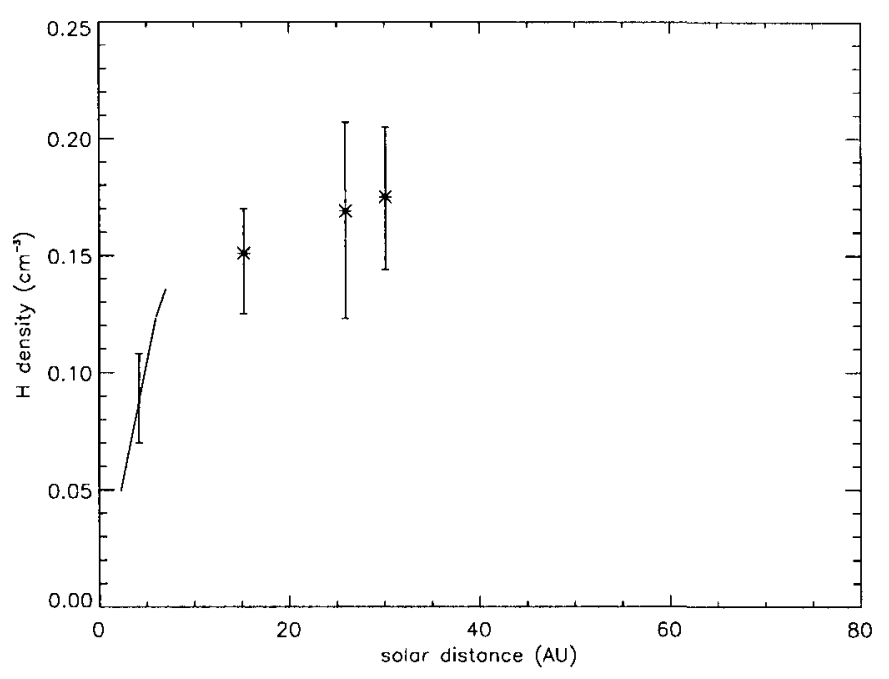

Fig. 1. H density distribution along Voyager 2 path derived from Lyman- $\alpha$ sky maps using the invariance principle.

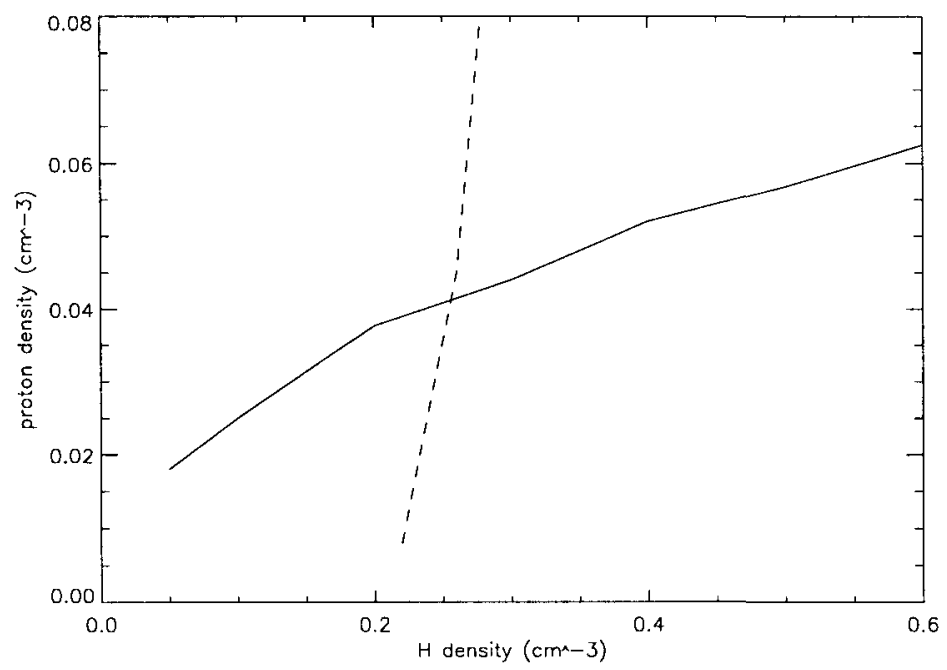

Fig. 2. Relations between the LIC proton and $H$ neutral densities derived from the connection of the LIC to the Voyager $\mathrm{H}$ distribution (see Fig. 1) through Baranov model (dashed curve) and from the assumption of a local ionization equilibrium, taking $\Gamma_{\mathrm{H}} \simeq 2.510^{-15} \mathrm{~s}^{-1}$ and $\Gamma_{\mathrm{He}} \simeq 6.910^{-15} \mathrm{~s}^{-1}$ (solid line). The intersection gives the actual $\mathrm{H}$ and proton densities, $\mathrm{n}_{\mathrm{H} \infty} \simeq 0.24 \mathrm{~cm}^{-3} \mathrm{n}_{\mathrm{H}^{+}} \simeq 0.043 \mathrm{~cm}^{-3}$ respectively, in the local cloud. 
consistent way the following set of properties of the local cloud at $\sim 1000 \mathrm{AU}$ from the Sun:

- H neutral: $\mathrm{n}_{\mathrm{H}}=0.24 \pm 0.05 \mathrm{~cm}^{-3}$

- proton: $\mathrm{n}_{\mathrm{H}^{+}}=0.043 \pm 0.005 \mathrm{~cm}^{-3}$

- He neutral: $\mathrm{n}_{\mathrm{He}}=(2.7 \pm 0.5) 10^{-2} \mathrm{~cm}^{-3}$

$-\mathrm{He}^{+}: \mathrm{n}_{\mathrm{He}^{+}}=(1.5 \pm 0.3) 10^{-3} \mathrm{~cm}^{-3}$

- electron: $\mathrm{n}_{\mathrm{e}}=0.044 \pm 0.005 \mathrm{~cm}^{-3}$

$-\mathrm{He}^{++}: \mathrm{n}_{\mathrm{He}^{2+}}=(1.0 \pm .2) 10^{-5} \mathrm{~cm}^{-3}$

\section{Conclusion}

After extraction of the hydrogen distribution from the Voyager Ly- $\alpha$ sky maps inside the heliosphere (see Fig. 1) and assuming local statistical equilibrium, we derived that the LIC has a high $\mathrm{H}$ density $\mathrm{n}_{\mathrm{H} \infty} \simeq 0.24 \mathrm{~cm}^{-3}$ and is weakly ionized with an ionization fraction near the Sun of $\sim 15 \%$ and $\sim 6 \%$ for $\mathrm{H}$ and $\mathrm{He}$ respectively. The self-consistency of the method used here, makes the derived set of LIC parameters unique for the ionization sources considered here, which could however not explain the enhanced He ionization with respect to $\mathrm{H}$ found by Dupuis et al (1995).

In order to check the validity of the weak ionization inferred for $\mathrm{H}$, it is interesting to compare our electron density to available measurements. We apply a simple model that calculates the photoionization of $\mathrm{H}$ and $\mathrm{He}$ from the Sun out to the edge of the local cloud to different lines of sight. Toward $\epsilon$ CMa or Capella, we obtain a mean electron density $\left\langle n_{\mathrm{e}}\right\rangle \simeq 0.08 \mathrm{~cm}^{-3}$, which is in good agreement with recent HST measurements made by respectively Gry et al (1995) and Wood \& Linsky (1995). As far as the ionization of $\mathrm{H}$ is the main pool for electron production, this result seems to confirm that this process is well described by our model. Nevertheless, an additional source that preferentially ionizes He atoms is required to explain EUVE results (Vallerga 1996).

\section{References}

Baranov, V.B, Malama, Y.G. (1993): JGR, 98 A9, 15157

Dupuis, J., Vennes, S., Bowyer, S., Pradhan, A., Thejll, P. (1995): ApJ, 455, 574

Gry, C., Lemonon, L., Vidal-Madjar, A., Lemoine, M., Ferlet, R. (1995): A\&A, 302, 497

Puyoo, O., Ben Jaffel, L., Emerich, C. (1997): ApJ, 480, 262

Slavin, J.D. (1989): ApJ, 346, 718

Vallerga, J. (1996): Space Sci. Rev., 78, 277

Vallerga, J., Welsh, B.Y. (1995): ApJ, 444, 702

Wood, B., Linsky, J. (1997): ApJ, 474, 39 